\title{
Computability of Emotions
}

\author{
Soaad Q. Hossain \\ Department of Computer and Mathematical Sciences, \\ Department of Philosophy \\ University of Toronto
}

\begin{abstract}
In the Philosophical Investigation, Ludwig Wittgenstein suggested that emotion is nothing but behavioral expressions through an open-ended sequence of actions. Edmund Husserl made a claim in Logical Investigations saying that pure mathematics would be made a branch of psychology as mathematics, specifically adding, subtracting, dividing and multiplying, are just a result of mental processes. Combining the suggestion and claim made by these two philosophers and using information process theory, cognitive appraisal theory, behaviorism and number theory, this paper will argue that emotion is algorithmic by showing the computability of emotion and how the equation for emotion can follow a logical sequence of actions that ultimately determines a specific emotion.
\end{abstract}

Keywords: emotion, algorithm, experience, memory, information, perception, pattern recognition, event, computability

\section{Introduction}

Psychology and mathematics are often considered different branches as psychology investigates behavior while mathematics uses numbers, quantity, and spaces, to formulate new conjectures. While the thought of having both branches intersect seems unlikely, it is not as unlikely as one would think. However, a philosopher by the name of Edmund Husserl claimed that they do intersect. Husserl discusses the idea of psychology and mathematics intersecting, going further as claiming that mathematics can be a branch of psychology (1). He uses the example of addition, subtraction, multiplication and division being simply mental processes in the brain. If one reversed this statement, the statement would then say that psychology can be a branch of mathematics. However, psychology is much more abstract and less certain than mathematics. Within psychology, there are some concepts that are less abstract than others. Some would say emotion is a concept that is not abstract and can be determined. Wittgenstein was one of the first individuals to suggest that emotion is just a sequence of actions. Emotion is a result of mental process that is simply a result of selected arguments, computed together resulting in a specific emotion. With the rise of artificial intelligence, the discussion of the computability of emotion is important as the scientific and philosophical community is in search 
for this. In this paper, I will first demonstrate that psychology can be a branch of mathematics, moreover, arguing how emotion is not only computable, but is also algorithmic.

\section{Computation of Mathematics}

Mathematics is abstract in the sense that it deals with many intangible subjects which includes numbers and operations. For example, mathematics covers the operation of adding things together, such as adding two arguments together. The first argument would be one number, the second argument would be another number, and so on. It is important to note that enough information must be provided to mathematically compute and provide an output. When looking at the case of emotion, it is quite challenging to determine the arguments that would calculate emotion. However, using Husserl's logic of mathematics being a possible branch of psychology, we find that there must be some conditions that must be met prior to any sort of mental process taking place. The conditions that must be met are: 1) having knowledge and 2) understanding of theoretical and symbolic laws. In the example of Husserl, for one to perform a mental process of addition, subtraction, multiplication and division, one must have prior knowledge in mathematics, specifically mathematical laws (1). One that does not know how to add numbers will not be able to have such mental processes that Husserl alludes. The prior knowledge plays a crucial role here because without the prior knowledge, no mental process of the sort can take place.

When viewing the computation of mathematics performed by an individual, we find that while the core of the problem relies on the information provided, the overview of the problem relies on two kinds of knowledge: prior (general information given beforehand, also known as a priori) and posterior (specific information given after, and considered, also known as a posteriori). Prior knowledge allows one to understand and comprehend while posterior knowledge allows one to do something with the information that one understood. There is, however, a condition that is required to have both the prior and posterior knowledge properly utilized, and that is the given information (i.e. the information provided). When a person is given the function $1+1=$, a person uses his or her prior knowledge to understand the question and to recall his or her knowledge with respect to addition, such as recall the definition of addition, then uses his or her posterior knowledge to use the two arguments (the 1s) and add them together to find the value of the function, with the value being the output of the function. The prior must come before the posterior, or else the sequence of actions required to get the right solution would be lost. If we were to do the function $1+1=$ without understanding the concept of adding, then regardless of how hard one tries to answer this question, one will not be able to find the answer. Similarly, if one understands how to add, but views the function $1+1=$ and does not understand 
what is asked, then one will not be able to provide the solution to that problem. The order of when actions take place matters.

The order of which the operation of mathematics take place follows the following sequence of actions: representation, identification, memory, recognition, process, and value. The first step to the sequences of actions is representation. Information provided to us is always in some method or form of communication. In the case of mathematics, most cases involve the information being provided visually. The way the information is presented heavily influences the way the information will be understood. For example, someone that does not know or understand the concept of PEMDAS (parentheses first, followed by exponents, multiplication and division, lastly addition and subtraction) will understand and evaluate a function such as function $4+(6-5) \times 2^{2}=$ differently compared to someone that does understand PEMDAS. The second step involves identification. Identification involves the individual identifying key components of the information that will help them understand things. Using the same function as before, a person can identify the numbers and operations within the function. Once the numbers and operations are identified, the person can move onto the third step, which involves memory. Unlike other steps, this step requires the most sub-steps compared to others.

\section{Memory and Mathematics}

In memory, there are key components in the memory capacity of a person that plays a key role when it comes to remembering something. A simple way of understanding memory is that memory is the process of maintaining information over time. In the case of mathematics, the first thing a person must remember is whether the person has seen the operations before. Once the person can remember encountering the operation, the person must then remember what operation does. The person must recall that the operation ' + ' adds the two arguments together while the operation '-' subtracts two arguments together. The person must then remember the order of which operations should be executed (remember PEMDAS). This would lead to the step of recognition. Recognition involves accepting what you see, accepting the fact that you recognize what you see and accepting that you understand what you see. The completion and combination of memory and recognition will then allow a person to process the information provided. In the case of mathematics, this is the process where a person calculates what the value is given a set of arguments and operations. Once the person completes his or her calculation, with the use of memory, they are then able to tell and provide the correct value.

The complexity of memory is quite complicated when it comes to the steps required to recall something. Interestingly, the complexity of memory and the order of which mathematics takes place can be effectively narrowed down. To effectively narrow the actions necessary to complete mathematical problems, such as $4+(6-$ 
5) $\times 2^{2}=$, one can narrow down the actions as follows: perception, experience, process, and value. Perception is the process of identifying, organizing and interpreting information to understand the information that is being presented. In the case of mathematics, a person's perception would first view the given information that is presented. According to Husserl, this would involve a person to perform either a phenomenological or objective analysis (1). The individual can choose to approach the problem using phenomenological analysis or objective analysis. The phenomenological analysis involves the individuals having a conscious experience of encountering either the exact same mathematical problem $\left(4+(6-5) \times 2^{2}=\right)$ or something similar. Phenomenological analysis heavily utilizes recognition, pattern recognition or both to identify an object. Once the object is identified, it can then be organized, allowing for easy interpretation. Objective analysis involves not being influenced by external factors, such as personal feelings or opinions, when considering the function $4+(6-5) \times 2^{2}=$. Objective analysis heavily utilizes the given facts and information on the subject at hand rather than taking his or her thought and opinions along with other aspects into consideration. Solving the $4+(6-5) \times 2^{2}=$ would ideally require both phenomenological analysis and objective analysis as one would first identify the problem using pattern recognition, then organize it and interpreting it by using only the information provided, ignoring external factors altogether.

The crucial part to perception is memory because perception utilizes memory for pattern recognition. As stated above, memory is the process of maintaining information over time. However, given the context of recognition and pattern recognition, memory can be seen as the means by which one would draw past experiences and use that information in the present. When memory is used in mathematics, specifically mathematical problems such as $4+(6-5) \times 2^{2}=$, it would be used to not only remember theories, laws and concepts, but also moments when one encountered the same or a similar mathematical problem. Pattern recognition helps recognize things such as operations and functions easily. Once one can recall doing a similar mathematical problem to $4+(6-5) \times 2^{2}=$, such as $4 \times(6-5)+2^{2}=$, one is then able to remember their experience encountering that mathematical problem. The progressive formations of presentations of prior mathematical problems form single experiences (1). For example, the mathematical problem $1+1=$ and $4 \times(6-5)+2^{2}=$ will formulate a total of two single experiences. The combination of these two single experiences into one single experience, which Husserl calls the "unity of experience" (1). The combination of perception and the unity of experience will ultimately lead to the final step, the process.

\section{Mental Processes}

According to Husserl, the process step is merely mental processes (1). Process is the procedure necessary to obtain an output. For the case of mathematics, 
we use it to find the value. We use the information provided (representation of the problem), our perception of the represented information, and our memory relating to the represented information. Once we combine these, we are then able to determine what would be the best approach and method to solve the mathematical problem. For the case of $4+(6-5) \times 2^{2}=$, one can use an inductive method and number theory (arithmetic), which involves observing, learning from experience and applying the experience that one has learned. After observing the information and recalling the operation order of PEMDAS, one will then be able to have a mental process and solve and compute $4+(6-5) \times 2^{2}=$, giving 8 as the desired value.

While there are countless psychological mental processes that took place during the process step, the mental processes that were possibly more noticeable than the others were perception, pattern recognition, and experience. The sum of these mental processes output a value. This shows that psychology can be a branch of mathematics as in psychology, mental states are created as outputs that resulted from combining and computing multiple mental processes together. Mental processes can utilize mathematical theories to help accurately determine the final mental state or mental process of a person.

\section{Computing for Emotion}

There are many kinds of mental processes in psychology. One of these many processes is emotion. If emotion is a mental process and mental processes can utilize mathematical theories to help accurately determine the final mental state or mental process, then that implies that emotion can utilize mathematical theories to help accurately determine the final emotion of a person. This would ultimately lead to the computation of emotion. The question would then arise of what kind of mathematical theory and what arguments would be used to help determine emotion. The solution to this question comes from three theories: number theory, information

process theory, and cognitive appraisal theory. All three theories utilize what has been mentioned earlier: arithmetic, interpretation, memory, perception, representation (or provided information) and pattern recognition. For representation, in the case of an emotion, it would simply be an event taking place. For pattern recognition, to occur, memory is required. Memory englobes pattern recognition. This simplifies things to: arithmetic, memory, perception and event.

Despite emotion being a mental process and perception playing an important role in the computation of emotion, this paper will not include or investigate perception because both these require the involvement of neuroscience and neurophysiology to determine the internal activities in the brain that contribute to the determination of emotion. When the brain is involved with emotion, there are certain internal activities in the brain that need to be taken into consideration. Regardless of how short the presentation of information (in this case an event) is presented, signals inside the brain relating to the presence of an event are made 
available to several emotion-triggering sites [within the brain] and will activate within the brain's sensory processing system (2). A perfect example of this is art. Art reflects the inner life of an artist by mirroring emotional states through displaying creativity, experience, psychology, and cognition (3). If one were to look at a painting, the region of the brain that deals with emotion and cognitive features would immediately trigger, working on what kind of emotion will be determined. This shows that there is a developmental sequence to emotion (3). While this can be implemented within the argument, to avoid discussing this complex matter, perception will not be included within the simplification of the argument behind the computability of emotion. This would require the use of perception, which we are trying to avoid discussing due to its complexity. For that reason, the explanation regarding the computability of emotion will solely incorporate number theory, information process theory, memory and event, and will occasionally refer to perception. The removal of perception leaves memory, event, information process theory, cognitive appraisal theory, and number theory left. Given this information, using information process theory, cognitive appraisal theory and number theory, I would like to propose and explain an equation, which essentially can be used to compute (solve the problem in an effective manner) and precisely determine emotion:

\section{Memory + Event $=$ Emotion}

The memory component of the equation connects heavily with information process theory. Information process theory is a psychological theory that uses a cognitive approach to understand how the mind of a human mind's transforms sensory information. It utilizes three kinds of memories: sensory memory, shortterm memory and long-term memory. Sensory memory is quicker than short-term memory as it relies on retaining memory through stimuli (within the moment an event occurs) and requires an event to take place to sense the stimuli. The information is attained through senses (sight, hearing, smelling, tasting and touching). Once the event has occurred and the information is gained, it can then be either perceived or ignored. In sensory memory, an emotion is noticed as a reaction to the event. For example, if one were to go through the event of feeling pain through nightmare for the first time, the person can either perceive the feeling of pain, or ignore it like it had never happened. If the information of the nightmare is ignored, then the person will likely forget about the feeling of pain within a set amount of time. However, if the person decides to perceive the information after the nightmare has occurred, then that information becomes sensory information.

\section{Emotion Equation Analysis}

Collective amounts of sensory memory (by having experienced multiple different occurring events on the same matter) can create recognizable patterns, 
leading to short-term memory. It is important to understand that once information has successfully passed the sensory memory and has reached the short-term memory stage, that information is understood and can be seen and recalled without the event physically taking place at the specified moment to the point where one can say that they describe it within at least a few words or sentences (4). The description would generally be able to associate an emotion with the event. For example, in the nightmare one had, one can describe the nightmare and associate it with fear, causing him or her pain. Sensory information catches one's attention, and thus progressing into short-term memory. Upon reaching the short-term memory, this is where memory becomes either one of two things: remembered or forgotten. Short-term memory (also known as working memory) is the retention of small amounts of information over brief time intervals (5). Short-term memory is influenced mainly by significance, utility, and repetition. Memories that are significant and relevant will tend to be remembered during short-term memory as it will be used later. Similarly, useful and necessary information tends to be remembered during short-term memory. During short-term memory, emotion is properly associated with events. It is possible for significant and useful information to be forgotten, but requires a certain amount of time to forget the information. The complex case comes when repetition is involved. When similar events occur multiple times, it becomes quite difficult for one to forget the memory. Alternatively, it becomes easier for one to remember the memory. In general, if it rains every other day of the week every week, it would be quite difficult for one to forget the sense of rain and their reaction to rain, and easier for him or her to recall and remember it. In short-term memory, there is one additional factor that plays a role in short-term memory.

The additional factor that plays a key role in short-term memory that was shortlisted is the amount of mental effort that one can engage in at the moment of an event. When it comes to mental effort, both individual characteristics and intellectual capacities are actively involved in mental effort. Similar to the case of perception, both individual characteristics and intellectual capacities rely and require the involvement of neuroscience and neurophysiology to determine the internal activities in the brain that contribute to the determination of mental effort engagement. Nonetheless, this factor along with the mentioned factors all contribute to short-term memory of an individual. From collecting numerous memories (through sensory memory and short-term memory), selected memories and moments are saved and stored as long-term memory. Long-term memory is different from sensory memory and short-term memory because long-term memory englobes memories such as on procedural memory, semantic and episodic memory, and mental imagery.

The first type of memory in long-term memory is procedural memory. Procedural memory is simply the memory one uses to perform procedures. For example, in order to read, write and communicate, one must know a language. In 
all languages, there is an order and structure to how words and sentences are said and written. Procedural memory would essentially be used to help a person understand how to use words and where to use them. In the context of emotion, procedural memory would associate what kind of emotion to use and when to apply that emotion. If we use the mathematical problem from earlier, procedural memory would be used to compute $4 \times(6-5)+2^{2}=$ by recalling, understanding and applying the order and rules associated with PEMDAS.

The second type of memory in long-term memory is semantic and episodic memory. Semantic and episodic memory are two different kinds of memories. Semantic memory is the memory one uses to record facts, statements, meanings (including written and verbal symbols), concepts, and knowledge of things. For one to remember what the modal model of memory (a model that assumes that information is received processed, and stored differently for each kind of memory)(6), then one would use his or her semantic memory to recall and remember this model. Similarly, one would use semantic memory to understand what operations are required to solve the mathematical problem $4 \times(6-5)+2^{2}=$. Episodic memory is the memory associated events that occurs in one's life. It is the memory one uses when recalling experiences and specific events in such a manner that allows one to recreate the experience and event that took place at given point of one's life. With episodic memory, one can recall and remember the time, place, emotion and other contextual knowledge of an event that occurred in his or her life. For example, one can remember the last time they had an Espresso coffee, where they had it, when they had it, and the taste of the coffee (which would be complimented with the emotion they would have towards the Espresso coffee). The combination of these information allows him or her to view a visual representation Espresso coffee in their mind without the coffee physically being present. Visual representation then leads to the third and final type of memory in long-term memory is mental imagery.

Mental imagery (or simply imagery) can be understood as the representation of something in mind. Imagery can use sensory memory, semantic and episodic memory to generate visual representation of objects. Through pattern recognition, imagery helps one with recalling and remembering specific information. For instance, if one wants to remember what a painting looked like, one can use imagery to mentally visualize what the painting looks like without the painting physically present in front of him or her. An emotion is generally associated with the visual representation as a sort of reaction to the visual representation. Imagery along with the other types of memories mentioned earlier are all used in the equation Memory + Event $=$ Emotion .

In the equation Memory + Event $=$ Emotion, the combination of sensory memory, short-term memory, and long-term memory results in the "Memory" argument in the equation, leaving the remaining argument "Event". Different from 
the memory argument, I find that event does not involve information process theory at all, but rather it involves behaviorism and cognitive appraisal theory. There are two definitions to the term behaviorism, one being philosophical while the other being psychological. For the purpose of the equation and context, we will be using the psychological definition of behaviorism. In psychology, behaviorism is essentially the systematic approach of understanding people's response or behavior to certain stimuli in the environment. This definition of behaviorism works perfectly with the argument "Event" as there is a direct connection between behaviorism and event, which that connection is the event itself. The cognitive appraisal theory is about one's personal interpretation of an event in determining their emotion in proportion to the event. In the equation, an event is simply not just an event taking place, but also a person's reaction to that event. This takes the context of behaviorism and cognitive appraisal theory as it takes the response, specifically the emotional response, a person has to an event. For example, if one sees a scary movie, then the person's emotional reaction to that movie is feeling scared. Similarly, if one sees a child that is happy, he or she might have an emotional response of feeling joy. The essential point to note about the argument "Event" is that it is one's emotional response to the current event taking place.

Given both the description of memory and event, in the equation Memory + Event $=$ Emotion, I will now explain how the computation process of the equation works. To begin, I will first investigate the argument "Memory". The argument memory would be the memory one has of all his or her events that they have experienced in their life. The addition operator will then add these memories to the argument "Event", which takes the person's emotional response to the current event taking place to ultimately determine which emotion will be outputted from the equation. The complex part of the equation comes from the fact that both arguments influence each other either directly or indirectly. One's reaction to an event is influenced by one's memory of the exact same event or a similar event. Similarly, one's memory of an event comes from a memory of himself or herself experiencing and previous reactions to that event or a similar event. This complexity implies that it satisfies the law of addition in number theory, which is:

$$
\text { Memory }+ \text { Event }=\text { Event }+ \text { Memory }=\text { Emotion }
$$

No matter which argument comes first, then equation still holds. The first memory of an event is managed by sensory memory resolves the issue of wondering what would happen if a person does not have any sort of memory of encountering a specific event before. Conditions for both arguments are satisfied through the differences one argument has to offer compared to the other argument. The sum of these differences as well as the complimentary result in an emotion being determined. Visualizing the equation using a painting (as the event encountered) a person is scared of. If it is the first time one is looking at the painting, then the equation would look like: 


\section{Painting + Memory $=$ Fear}

If it is not the first time one is looking at the painting, then the equation would look like:

$$
\text { Painting }+ \text { Memory }=\text { Fear }
$$

Similarly, if it is not the first time one is looking at the painting and has prior memories of looking at paintings, then the equation would look like:

$$
\text { Painting }+ \text { Memory }=\text { Fear }
$$

The interesting part in this equation is that not only can we notice that the equation satisfies the conditions of number theory and is computable, but it can also become algorithmic. Algorithm is a step-by-step procedure used to solve a problem (7). I find that emotion can be algorithmic but will be quite complex as there would be multiple if and then statements along with other kinds of statements that would be used to solve for the emotion.

\section{Conclusion}

There is certainly a method to compute emotion. The equation Memory + Event $=$ Emotion is a possible approach I propose to compute emotion. I find that if we properly understand and combine the concept of memory (through the perspective of information process theory) and event (through a behavioristic perspective), we can effectively compute emotion. The next step in investigating the possibility of computing emotion would be to further investigate the mathematical and psychological arguments, and consider the neuroscience and cognitive neuroscience components, specifically dealing with inner activities in the brain in regard to perception. Also, given that psychology can be viewed as a branch of mathematics, this shows us that not only is emotion in psychology computable, but other areas in psychology can be computed as well. This can lead to more possible research and discoveries in computational psychology, cognitive science and cognitive psychology.

\section{Acknowledgement}

There is certainly I would like to thank Karolina Hübner (Cornell University) for useful discussions and suggestions. This manuscript has been released as a pre-print at PsyArxiv (8).

\section{Conflict of Interest}

Conflict of Interest The author declares no conflict of interest in preparing this article.

\section{Funding}


The author received no financial support for the research, authorship, and publication of this article.

\section{References}

1. Husserl E. Logical Investigations. Routledge, 1970, p. 178 - 179, 207, 349.

2. Parasuraman R., and Rizzo M. Neuroergonomics, The Brain at Work. Oxford University Press, 2007, p. 179 - 181.

3. Zaidel D. W. Neuropsychology of Art, Neurological, Cognitive and Evolutionary Perspectives. Psychology Press, 2005, p. 171 - 175.

4. Wittgenstein L. Philosophical Investigations. 3rd ed. Basil Blackwell Ltd., $1958,105^{\mathrm{e}}$.

5. Tulving E., and Craik F. I. M. The Oxford Handbook of Memory. Oxford University Press, 2000, p. 77 - 80.

6. Galotti K. M.,Fernandes M. A., Fugelsang J., and Stolz J. A. Cognitive Psychology, In and Out of the Laboratory. Sage Publishing, 2009, p. 140 - 143.

7. Neapolitan R. E., and Naimipour K. Foundations of Algorithms Using Java Pseudocode. Jones and Bartlett Publishers Inc., 2004, p. 2 - 3.

8. Hossain S. Computation of Emotion. PsyXiv, https://doi.org/10.31234/osf.io/guafc (2020 accessed 11 August 2020). 